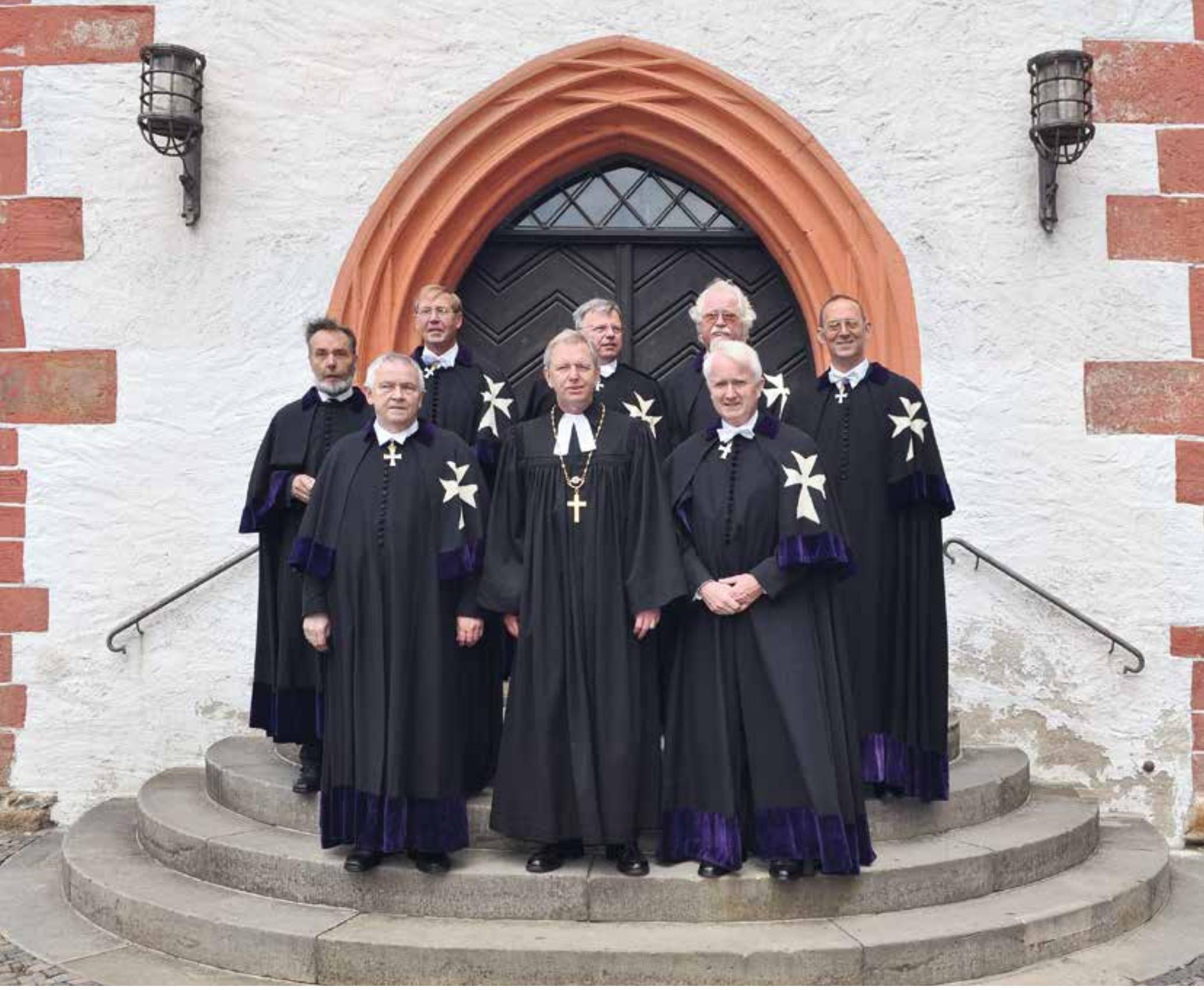

\title{
Das Domstift St. Marien in Wurzen
}

\section{Leo von Sahr-Schönberg}

Im Jahr 1114 gründete Bischof Herwig von Meißen das Kollegiatstift St. Marien in Wurzen, das nunmehr über 900 Jahre alt ist. Das Wurzener Domstift unterscheidet sich erheblich von einer Stiftung, die auf ewig angelegt ist und aus dem Stiftungsvermögen den Stiftungszweck erfüllen soll. Aber auch im Vergleich zu den drei anderen Domstiften in Meißen, Naumburg und Brandenburg sind wir anders aufgestellt und vor allem auch organisatorisch und finanziell deutlich kleiner ausgestattet.

\section{Rechtsform}

Das Domstift Wurzen ist ein rechtsfähiges evangelisch-lutherisches Stift der Evangelisch-Lutherischen Landeskirche Sachsens mit Sitz in Wurzen.
Die gesetzliche Vertretung und Verwaltung des Domstifts erfolgt durch das Domkapitel. Das Domkapitel ist eine Körperschaft des öffentlichen Rechts in der sächsischen Landeskirche. Stiftsherr ist der jeweilige Landesbischof. Außer dem Kustodiegebäude in Wurzen hat das Domstift kein eigenes Vermögen. Der Jahreshaushalt hat nur ein Budget von rund 40.000 Euro. Die Verwaltung besteht aus einer Verwaltungsangestellten mit einer 11-Prozent-Stelle und einem Domküster mit einer 10-Prozent-Stelle. Die notwendigen Investitionen muss ich als Dechant beim Landeskirchenamt gesondert anmelden. Insofern ist das Wurzener Domstift nicht nur klein im Vergleich zu den anderen Stiften, sondern auch innerhalb der sächsischen Landeskirche eine Rarität.
Das Wurzener Domkapitel mit Landesbischof Jochen Bohl, 2014 


\section{Die Köpfe des Domkapitels}

Da das Domkapitel mit einer minimalen Verwaltung auskommen muss, werden die sieben ehrenamtlich tätigen Domherren weniger nach ihrer Stellung in der Gesellschaft, sondern vielmehr nach ihrer jeweiligen Sachkompetenz ausgesucht. Neben dem Dompropst (dem erfahrenen Superintendenten i. R. Christoph Richter) und dem Dechanten besteht das Kapitel aus dem Senior, dem Subsenior und weiteren drei Kapitularen. Weibliche Mitglieder haben wir derzeit noch nicht; diese würden satzungsgemäß als Kapitularinnen bezeichnet. Das Domherrenamt wird bis zum 75. Lebensjahr ausgeübt; danach wird man Altdomherr und bringt sich üblicherweise - dann ohne Stimmrecht - weiter für den Dom ein. Die einzelnen Domherren sind ausgewiesene Experten auf ihren Fachgebieten, wie z.B. Dr.-Ing. h.c. Eberhard Burger, der sich für die baulichen Belange einsetzt (der aber in Kürze Altdomherr wird) oder Heinz Hartwig Böhmer, der als Vorsitzender des Finanzausschusses der Synode seine Erfahrung in Finanzfragen einbringt. Der Bereich der Musik wird abgedeckt vom Kirchenmusikdirektor und Domkantor Johannes Dickert. Und genauso sind die Bereiche der Religionswissenschaften mit Prof. Dr. Wolfgang Ratzmann und der juristischen Angelegenheiten mit OLKR i. R. Dietrich Lenk sehr kompetent besetzt. Und ohne das Engagement unseres Altdomherrn Superintendenten i. R. Horst Schulze gäbe es den Wurzener Dom nicht mehr; die Landeskirche wollte das Gebäude in den 1980er Jahren aufgeben.

\section{Aufgabenfelder}

Die Tätigkeitsfelder des Domkapitels erstrecken sich auf vier Felder, auf denen wir uns zwischen Beständigkeit und dem Agieren als „moderner Player" versuchen können.

\section{Geistliche Arbeit}

Das Domstift St. Marien zu Wurzen hat keine eigene Kirchgemeinde, jedoch nutzt die Kirchgemeinde Wurzen St. Wenceslai den Dom für die Gottesdienste in der warmen Jahreszeit von Ostern bis zum Reformationsfest. Aus Sicht eines Dechanten ist die Kirchgemeinde sozusagen der größte Kunde des Domkapitels. Einmal im Jahr, in der Regel nach Trinitatis, findet der Domherrentag statt, den wir mit einem feierlichen Gottesdienst und einer Predigt unseres Stiftsherrn, dem Landesbischof, begehen. Die Theologen unter den Domherren übernehmen im Laufe des Jahres immer wieder Gottesdienste. Der Dom ist auch beliebt für Trauungen und Taufen. Die geistliche Arbeit des Domkapitels würde ich somit eher unter den Begriff der Beständigkeit einstufen.

Autor

Leo v. Sahr-Schönberg

Wurzen chenmusik als auch die Musik in der Kirche. Bis zu zwanzig Musikveranstaltungen finden jedes Jahr im Dom statt, die ungefähr 3.000 Besucher anziehen. Der Dom bietet 600 bis 700 Zuhörern Platz. Der Schwerpunkt liegt auf der klassischen Musik, aber es sind auch immer wieder andere Musikrichtungen im Dom zu hören. Musikalisch hat der Dom überregionale Bedeutung. Ein Schwerpunkt liegt auch auf der Kinder- und Jugendarbeit. Der Domkantor unterrichtet wöchentlich im Kindergarten und häufig sind Kindergartengruppen im Dom, denen u. a. die Orgel erklärt wird. Sowohl die Vielfalt als auch der Umfang der kirchenmusikalischen Arbeit äußern sich in den diversen Chören und Orchestern: Jugendkantorei, Wurzener Kurrende, Capella Sancti Wenceslai, Domkantorei, Kammerorchester und Posaunenchor. Zusätzlich nutzen mehrere Organisten die Jehmlich-Orgel, die die größte Orgel im Muldental ist.

\section{Kulturelles Leben}

Den Dom - neben der Musik - mit kulturellem Leben zu erfüllen, ist für ein ehrenamtlich tätiges Domkapitel, das zudem überwiegend nicht vor Ort wohnt, eine Herausforderung. Man tut, was man kann. So versuchen wir in erster Linie den Dom als offenes Baudenkmal mit seiner wunderbaren Ausstattung zur Geltung zu bringen. Zur Ausstattung gehören auch die nicht unumstrittenen, aber ausdrucksstarken Bronzefiguren von Georg Wrba (1872-1939) aus den frühen 1930er Jahren. Es finden immer wieder Führungen statt. Auch für Vorträge und Empfänge wird der Dom genutzt. 2014 fand anlässlich der 900-jährigen Domweihe ein vielbeachtetes Kolloquium statt. Und im Jahr 2018 tagte die Arbeitsgemeinschaft für Sächsische Kirchengeschichte anlässlich ihres 70-jährigen Bestehens im Dom. Im Wurzener Dom sind auch immer wieder Wandertouristen zu sehen, da der Dom den Vorteil hat, dass er sowohl am Jakobspilgerweg als auch am Lutherweg liegt. Das Domkapitel arbeitet gut mit dem als Hotelrestaurant genutzen benachbarten Schloss (ehemaliger Bischofssitz) zusammen, da beide Gebäude ein einzigartiges Ensemble bilden.

\section{Bautätigkeit}

Ein Dom ist nie fertig, und ständig muss etwas restauriert werden. Die Investitionsliste wird jährlich überarbeitet; der für die Bauangelegenheiten zuständige Domherr erhält Unterstützung vom Baupfleger des Regionalkirchenamtes und der Dechant regelt die Finanzierung. Wichtig sind auch die Investitionen in den Erhalt der Kunstgegenstände, d. h. die Restaurierung von Bildern, Epitaphen und Büchern. Die Denkmalpflege und die Referate des Landeskirchenamtes wollen eingebunden und die Handwerker wollen gebeten sein. Die Erfahrung lehrt, dass ein über 900 Jahre alter Dom einfach Zeit braucht. Mal schnell etwas restaurieren zu wollen, funktioniert nicht. Auch „moderne Player“ werden hier eingebremst und lernen schon bald: In der Ruhe liegt die Kraft.
Musik

Eine wesentliche Kernkompetenz des Wurzener Domes ist die Musik, das heißt sowohl die Kir- 